\title{
Cancer Research in Low- and Middle-Income Countries: Consortiums, Implementation Science and Healthcare Delivery
}

\author{
Benjamin O. Anderson, MD \\ Departments of Surgery and Global Health Medicine, University of Washington, Seattle, WA
}

Having caused over 8 million deaths worldwide in 2013, cancer is now the second leading cause of human death. ${ }^{1}$ While cancer is often identified as a high-income country (HIC) health problem, low- and middle-income countries (LMICs) account for $57 \%$ of cases and $65 \%$ of cancer deaths globally. ${ }^{2}$ The burden of cancer will continue to shift to less-developed countries due to the growth and aging of world populations, with increasing prevalence of known risk factors. ${ }^{3}$ Looking ahead, the 12.7 million cases that occurred in 2008 are predicted to rise an additional $75 \%$ to 22.2 million by 2030 , the majority in LMICs. ${ }^{4}$

Cancer demographics vary among countries but correlate with the level of economic development. ${ }^{4}$ Cancers of the breast, lung, colorectum, and prostate account for half of the overall cancer burden in regions of the highest Human Development Index (HDI), while cancers of the esophagus, stomach, and liver are relatively more common in medium-HDI regions. Back in 1990, cervical cancer was more common than both breast and liver cancer in lowHDI regions but those trends are shifting. Cervical cancer prevention is improving as breast cancer rates continue to rise. ${ }^{5}$ Overall, the rapid societal and economic transition in many countries means that any reductions in infection-related cancers (cervical, liver, stomach) are offset by an increasing number of new cases that are more associated with reproductive, dietary, and hormonal factors (breast, colorectal, prostate) ${ }^{4}$

Global cancer consortiums (GCCs) can be defined as groups with a shared mission and common set of objectives that collaborate to advance some broad aspect of global

(C) Society of Surgical Oncology 2016

First Received: 9 September 2016; Published Online: 15 November 2016

B. O. Anderson, MD

e-mail: banderso@uw.edu cancer control. ${ }^{6}$ GCCs provide an overarching framework for advancing cancer control through defined common goals and/or evidence-based strategies. Some GCCs may address a specific global task, such as genomic data collection and/or cancer tissue banking at the global level. For example, the International Cancer Genome Consortium (ICGC) has declared its unifying goal to obtain a comprehensive description of common genetic changes in common tumors of clinical and societal importance across the globe. ${ }^{7}$ Other GCCs may focus on particular needs, such as the establishment of a platform for performing cancer research in limited resource settings. Consortiums are the foundation of collaboration.

\section{ESTABLISHMENT OF A CANCER RESEARCH CONSORTIUM IN SUB-SAHARAN AFRICA}

In this issue of Annals of Surgical Oncology, Fischer and colleagues describe the formation of a successful cancer research consortium in sub-Saharan Africa. ${ }^{8}$ Drawing on their experience of founding the African Research Group for Oncology (ARGO), which linked six academic teaching and healthcare facilities in Nigeria with the St. James University Hospital in the UK and the Memorial Sloan Kettering Cancer Center in the US, the investigators delineate steps and key factors needed for establishing a successful collaborative cancer research consortium. The group delineated four initial steps in establishing an LMIC-based cancer research consortium:

1. Identify institutional and individual collaborators.

2. Define an initial focused geographic scope (local, regional, or national).

3. Prioritize a common cancer where realistic improvements can be achieved.

4. Select research questions of common interest among the collaborators. 
The ARGO cancer research consortium began as a surgical collaboration but has now expanded to include nonsurgical collaborators. ARGO chose colorectal cancer as their initial clinical focus since this is a very common cancer amenable to surgical therapy about which little data exist in Nigeria. ARGO recognized that in establishing tempered, realistic goals, they needed to be guided by clinical relevance, resource constraints, and time-to-implementation limitations. They first chose to study cancer risk factors, presentation and recurrence patterns, and clinical outcomes as a basis for developing screening and/ or treatment trials.

Research team-building consists of integrating senior and junior collaborators to be integrated over a 3-year setup period to allow for analysis of current cancer needs and patient outcomes, completion of retrospective research projects, generation of prospective research project ideas, and application for funding opportunities. This period allows the collaborators to conduct exploratory analyses to identify research that is relevant to the local setting, and establishing research infrastructure such as metric establishment and data collection.

Formal assessment of key services, specialty expertise and infrastructure is an essential early step. Consortium agreements focused on research training can be essential since collaborators from LMICs may not have similar focus on research methodology and standards. For example, pathology services for tissue processing and interpretation are essential for both clinical management of cancer and cancer research infrastructure. The most successful efforts in building pathology services have used a combination of in-country training, improvements in pathology techniques, and remote assistance. ${ }^{9,10}$

In cases where equipment and services cannot be procured on site, sample shipping and processing methods can be established. The handling of fresh, fresh-frozen, and paraffin block tissues can be problematic, especially when tissues need to pass through customs and could be subject to delays. Laboratory equipment is often unavailable or in disrepair, impeding research progress. While some LMICs find innovative ways to adapt older equipment to the research environment, ${ }^{11}$ ARGO cautions against using substandard equipment, which can save on initial costs but may affect the validity of research results. In addition to the equipment itself, electricity can be an issue due to frequent power outages in some locations.

Research funding sources are limited, especially in global health, and need to be carefully considered by the consortium; however, research costs can be significantly lower in LMICs. In one study conducted by ARGO, colonoscopies for 100 subjects cost less than $\$ 15,000$, whereas in a similar study in the US, the cost would be up to $\$ 200,000$.

\section{IMPLEMENTATION RESEARCH AS A CATALYST FOR IMPROVING CANCER CARE IN LOW- AND MIDDLE-INCOME COUNTRIES}

In 2005, the 58th World Health Assembly (WHA) of the World Health Organization (WHO) passed a resolution urging member states "to establish or strengthen mechanisms to transfer knowledge in support of evidence-based public health and health-care delivery systems, and evidence-based health-related policies". ${ }^{12}$ There is value in studying cancer in LMICs where the distribution, extent of disease and environmental influences are fundamentally different from HICs, where most cancer research is performed. By establishing research protocols and creating the infrastructure necessary to operate them, the cancer research consortium becomes a vehicle for improving and systematizing clinical care.

Equally important to scientific investigation, there is a pressing need to understand how healthcare delivery systems can most effectively provide cancer detection, diagnosis, and treatment in limited resource settings. Implementation research focuses on translating clinical knowledge into programmatic development through scientific applications and policy strategies to promote systematic uptake of clinical research findings. ${ }^{13}$ Communicable diseases, such as HIV/AIDs, have achieved significant gains in health outcomes through effective implementation and quality improvement programs. ${ }^{14,15}$ By contrast, national and international initiatives in global cancer care were declared by the WHO almost 15 years ago, ${ }^{16}$ but only modest improvements in cancer outcomes have been achieved in LMICs despite increased health knowledge and expertise.

In cancer, targeted interventions can address the rising cancer burden through effective primary prevention strategies, vaccination, early detection, and effective treatment programs. ${ }^{4}$ Early detection and adjuvant systemic therapy are synergistic and mutually dependent for improving breast cancer outcomes since early detection only works if it can be followed by prompt, effective therapy. ${ }^{17}$ LMICs require an organized systematic approach, beginning with a comprehensive situation analysis. Resource-stratified guidelines provide a framework for analyzing healthcare delivery systems as a basis for improving patient outcomes, and may also frame valuable research questions that directly relate to the LMIC population being studied. ${ }^{18,19}$ The long-term strategy is to devise a comprehensive evidence-based plan that, with phased implementation, can successfully build functional and sustainable health systems. Cancer research consortiums can play an important role in correctly guiding this process by providing the essential evidence and data that are needed for making optimal health policy decisions. 
DISCLOSURE Dr. Anderson has no relevant conflicts of interest to report.

\section{REFERENCES}

1. Naghavi M. GBD 2013 Mortality and Causes of Death Collaborators. Global, regional, and national age-sex specific all-cause and cause-specific mortality for 240 causes of death, 1990-2013: a systematic analysis for the Global Burden of Disease Study 2013. Lancet. 2015;385(9963):117-71.

2. Torre LA, Bray F, Siegel RL, Ferlay J, Lortet-Tieulent J, Jemal A. Global cancer statistics, 2012. CA Cancer J Clin. 2015; 65(2):87-108.

3. Bray F, Moller B. Predicting the future burden of cancer. Nat Rev Cancer. 2006;6(1):63-74.

4. Bray F, Jemal A, Grey N, Ferlay J, Forman D. Global cancer transitions according to the Human Development Index (20082030): a population-based study. Lancet Oncol. 2012;13(8): 790-801.

5. Fitzmaurice C, Dicker D, et al. The Global Burden of Cancer 2013. JAMA Oncol. 2015;1(4):505-27.

6. Ilbawi AM, Anderson BO. Global cancer consortiums: moving from consensus to practice. Ann Surg Oncol. 2015;22(3):719-27.

7. International Cancer Genome Consortium. 2016. https://icgc.org/. Accessed 7 Sep 2016.

8. Fischer S, Alatise S, Komolafe A, et al. Establishing a cancer research consortium in low- and middle-income countries: challenges faced and lessons learned. Ann Surg Oncol. 2016.

9. Stalsberg H, Awuah B, Ibarra JA, Nsiah-Asare A. Re-establishing a surgical pathology service in Kumasi, Ghana: case report and discussion of barriers and key elements of a successful collaboration between low- and high-resource countries. Cancer. 2008;113(8 Suppl):2338-46.
10. Nelson AM, Milner DA, Rebbeck TR, Iliyasu Y. Oncologic care and pathology resources in Africa: survey and recommendations. $J$ Clin Oncol. 2016;34(1):20-26.

11. Harris E. Building scientific capacity in developing countries. EMBO Rep. 2004;5(1):7-11.

12. World Health Organization. World Health Assembly: resolution on health research. 2005. http://www.who.int/rpc/meetings/58th_ WHA_resolution.pdf. Accessed 7 Sept 2016.

13. Cazap E, Distelhorst SR, Anderson BO. Implementation science and breast cancer control: a breast health global initiative (BHGI) perspective from the 2010 Global Summit. Breast. 2011;20 Suppl 2:S1-2.

14. Price AJ, Ndom P, Atenguena E, Mambou Nouemssi JP, Ryder RW. Cancer care challenges in developing countries. Cancer. 2012;118(14):3627-35.

15. CanTreat International. Scaling up cancer diagnosis and treatment in developing countries: what can we learn from the HIV/ AIDS epidemic? Ann Oncol. 2010;21(4):680-82.

16. World Health Organization. National Cancer Control programmes: policies and managerial guidelines. 2002. 2nd ed. http://www.who.int/cancer/media/en/409.pdf. Accessed 7 Sept 2016.

17. World Health Organization Cancer Control Programme. Early detection of cancer. 2016. http://www.who.int/cancer/detection/ en/. Accessed 7 Sept 2016.

18. Harford J, Azavedo E, Fischietto M, Breast health global initiative healthcare systems panel. Guideline implementation for breast healthcare in low- and middle-income countries: breast healthcare program resource allocation. Cancer. 2008;113(8 Suppl):2282-96.

19. Carlson RW, Scavone JL, Koh WJ, et al. NCCN framework for resource stratification: a framework for providing and improving global quality oncology care. J Natl Compr Canc Netw. 2016;14(8):961-69. 